\title{
Derek Pletcher, Zhong-Qun Tian and David E. Williams (Eds): Developments in Electrochemistry. Science Inspired by Martin Fleischmann
}

\author{
Grégoire Herzog
}

(C) Springer-Verlag Berlin Heidelberg 2015

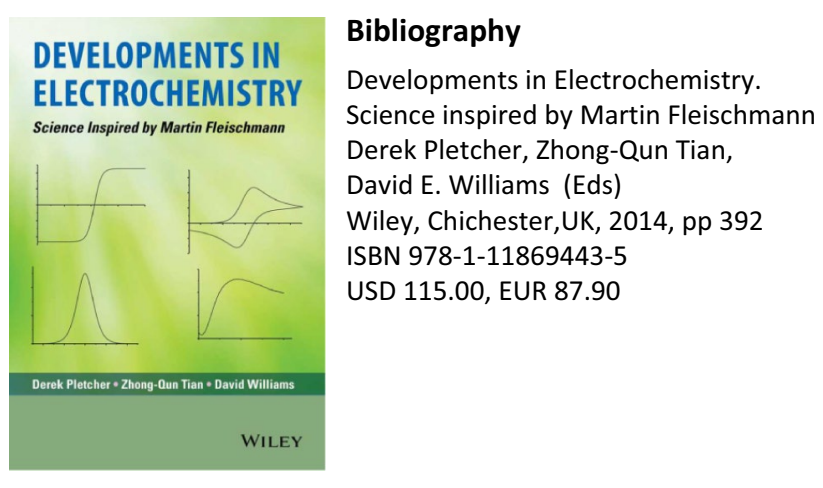

Development in Electrochemistry is a tribute to the scientific contribution of Martin Fleischmann (1927-2012) to the field of electrochemistry. Martin Fleischmann was a British electrochemist active in research over six decades (with his first paper published in the early 1950s and his last paper in 2009). Martin Fleischmann's name will always be associated with two major events in science in the last quarter of the 20th century, although both were of very different impact. The first one is the discovery of surfaceenhanced Raman spectroscopy in the mid-1970s. The second event is his participation to the 'cold fusion' scandal in 1989. Martin Fleischmann's scientific career cannot be reduced to these two separate events and this book contributes to the understanding of the extent of his influence on modern electrochemistry.

This book can be read as a whole as it shows how Fleischmann influenced and inspired a generation of scientists both in the United Kingdom and in the rest of the world.
Many of the contributors are linked to the University of Southampton, where he worked from 1967 to 1983 . His scientific legacy as described in this book is divided in 18 chapters. Each of these chapters is introduced by one paragraph on how the particular topic has been influenced by Fleischmann's research. One of the scientific research fields for which he was the most influential is the coupling of analytical techniques (SERS, STM, IR, and XRD) with electrochemistry for the in situ study of surface processes. Obviously, surface-enhanced Raman spectroscopy occupies a prime place with two chapters dedicated to it. In the 1970s, Fleischmann and his Southampton colleagues pioneered this field with the observation of pyridine adsorbed on a nanostructured silver electrode. His research also contributed to the development of electrochemical engineering and electrochemical methods (e.g., electrochemical impedance spectroscopy). It is almost impossible to write about Martin Fleischmann's scientific career without mentioning the 'cold fusion' scandal. This book is not shy on this regard with part of the biographical chapter and a full chapter with a positive view dedicated to it.

In the introduction, the editors claimed their intention to 'celebrate the legacy that (Martin Fleischmann) has left to electrochemistry' and that cold fusion 'should not be allowed to dominate our view of Martin Fleischmann as a remarkable and outstanding scientist'. The high quality chapters presented in this volume contribute greatly to achieving the editors' goal.

G. Herzog $(\bowtie)$

CNRS-Université de Lorraine, Lorraine,

Villers-lès-Nancy, France

e-mail: gregoire.herzog@univ-lorraine.fr 